\title{
Pulmonary veno-occlusive disease misdiagnosed as idiopathic pulmonary arterial hypertension
}

\author{
M. Palazzini and A. Manes
}

ABSTRACT: A 27-yr-old female with a 6-month diagnosis of idiopathic pulmonary arterial hypertension (PAH) confirmed elsewhere was referred to our centre with worsening dyspnoea.

On examination, the patient had low systemic oxygen saturation despite high oxygen flow and reduced exercise capacity. Haemodynamics were indicative of severe pre-capillary PAH. Highresolution computed tomography revealed diffuse ground-glass opacity with thickening interlobular septa, and haemosiderin-laden macrophages were identified by bronchoalveolar lavage. Based on clinical and diagnostic findings, the patient was re-diagnosed with pulmonary veno-occlusive disease (PVOD).

Treatment with high-dose diuretics and the endothelin-receptor antagonist bosentan improved the patient's exercise capacity, haemodynamics and quality of life. However, 1 yr later there was a progressive, slow deterioration in the patient's functional capacity and oxygen saturation, and inhaled prostanoid and oxygen therapy were initiated. Despite some subjective improvements, the patient's haemodynamics and oxygen saturation continued to decline and she underwent lung transplantation.

This case emphasises that PVOD is an under-recognised and often misdiagnosed form of pulmonary hypertension. Therefore, accurate diagnosis of PVOD requires comprehensive clinical and diagnostic work-up. While lung transplantation remains the treatment of choice for patients with PVOD, targeted therapies for PAH in addition to high doses of diuretics merit evaluation.

KEYWORDS: Pulmonary hypertension, pulmonary veno-occlusive disease

A 27-yr-old female was referred to the Pulmonary Vascular Diseases Centre of Bologna (Bologna, Italy) with a diagnosis of idiopathic pulmonary arterial hypertension (PAH) and a history of progressive dyspnoea during exercise since the age of 26 yrs. In August 2005, while overseas, she had been admitted to hospital for worsening dyspnoea. At this time she was in New York Heart Association functional class III and sarcoidosis was suspected. A transbronchial biopsy was performed but the sample was not sufficient for diagnosis. In September 2005, she was referred to a respiratory medicine department where sarcoidosis was excluded and idiopathic PAH was diagnosed. Diagnosis was confirmed 1 month later following referral to an expert pulmonary hypertension centre and treatment with high-dose sildenafil was initiated (80 $\mathrm{mg}$ t.i.d.). However, after 1 month of treatment, the patient exhibited worsening dyspnoea and was referred to our centre (Pulmonary Hypertension Unit, Institute of Cardiology, University of Bologna, Bologna) in February 2006.

On examination, the patient had right heart failure, low systemic oxygen saturation despite high oxygen flow $\left(88 \%\right.$ on $6 \mathrm{~L} \cdot \mathrm{min}^{-1}$ oxygen flow by nasal prongs), and reduced exercise capacity (6min walk distance (6MWD) $200 \mathrm{~m}$ ). ECG showed right ventricular hypertrophy with ventricular strain pattern. Her chest radiograph showed cardiomegaly, enlargement of the proximal pulmonary artery and diffuse interstitial oedema with basal Kerley B lines. Echocardiography confirmed dilatation of the right heart chambers and abnormalities in the shape and function of interventricular septum. Perfusion lung scans identified diffuse "patchy-like" sub-segmental hyperperfusion defects.
AFFILIATIONS

Pulmonary Hypertension Unit, Institute of Cardiology, University of Bologna, Bologna, Italy.

CORRESPONDENCE

M. Palazzini

Pulmonary Hypertension Unit

Institute of Cardiology

University of Bologna

Via Massarenti 9

40138 Bologna

Italy

E-mail: massimiliano.palazzini@tin.it

Received:

June 122009

Accepted after revision:

July 062009

PROVENANCE

Publication of this peer-reviewed article was supported by Actelion Pharmaceuticals Ltd, Switzerland (unrestricted grant, European Respiratory Review issue 113).

European Respiratory Review Print ISSN 0905-9180 Online ISSN 1600-0617 


\begin{tabular}{|c|c|c|c|}
\hline \multirow[t]{2}{*}{ TABLE 1} & \multicolumn{3}{|c|}{$\begin{array}{l}\text { Haemodynamic parameters on presentation and } \\
\text { following treatment with bosentan plus high } \\
\text { doses of furosemide }\end{array}$} \\
\hline & & Presentation ${ }^{\#}$ & $\begin{array}{l}\text { Bosentan plus } \\
\text { furosemide }\end{array}$ \\
\hline \multicolumn{2}{|c|}{ Heart rate beats $\cdot \min ^{-1}$} & 120 & 110 \\
\hline \multicolumn{2}{|c|}{ Right atrial pressure $\mathrm{mmHg}$} & 11 & 3 \\
\hline \multicolumn{2}{|c|}{$\begin{array}{l}\text { Pulmonary artery pressure } \\
\mathrm{s} / \mathrm{d} / \mathrm{m} \mathrm{mmHg}\end{array}$} & $98 / 50 / 70$ & $85 / 48 / 63$ \\
\hline \multicolumn{2}{|c|}{ Pulmonary wedge pressure $\mathrm{mmHg}$} & 10 & 8 \\
\hline \multicolumn{2}{|c|}{ Trans-pulmonary gradient $\mathbf{m m H g}$} & 60 & 55 \\
\hline \multicolumn{2}{|c|}{ Blood pressure $\mathrm{s} / \mathrm{d} / \mathrm{m} \mathrm{mmHg}$} & $102 / 76 / 90$ & $94 / 73 / 79$ \\
\hline \multicolumn{2}{|c|}{ Cardiac output $L \cdot \min ^{1}$} & 2.5 & 4.0 \\
\hline \multicolumn{2}{|c|}{ Cardiac index L $L \cdot \min ^{-1} \cdot \mathrm{m}^{-2}$} & 1.4 & 2.4 \\
\hline \multicolumn{2}{|c|}{ PVR $\mathrm{mmHg} \cdot \mathrm{L}^{-1} \cdot \min ^{-1}$} & 24 & 13.8 \\
\hline \multicolumn{2}{|c|}{ SVR $\mathrm{mmHg} \cdot \mathrm{L}^{-1} \cdot \mathrm{min}^{-1}$} & 31.6 & 19.0 \\
\hline \multicolumn{2}{|c|}{ PVR/SVR } & 0.8 & 0.7 \\
\hline \multicolumn{2}{|l|}{$\mathrm{Sa}, \mathrm{O}_{2} \%$} & 88 & 95 \\
\hline \multicolumn{2}{|l|}{$\mathrm{Sv}, \mathrm{O}_{2} \%$} & 39.1 & 60 \\
\hline
\end{tabular}

s: systolic; d: diastolic; m: mean; PVR: pulmonary vascular resistance; SVR: systemic vascular resistance; $\mathrm{Sa}_{1} \mathrm{O}_{2}$ : arterial oxygen saturation; $\mathrm{Sv}, \mathrm{O}_{2}$ : mixed venous oxygen saturation. ${ }^{\#}$ : oxygen, $6 \mathrm{~L} \cdot \mathrm{min}^{-1}$; : breathing room air.

Right heart catheterisation (RHC) confirmed severe pre-capillary pulmonary hypertension with a reduced cardiac index and reduced systemic and mixed venous oxygen saturation (table 1). Pulmonary wedge pressure was normal. Contrast enhanced computed tomography of the chest excluded chronic thromboembolic pulmonary disease, and high-resolution computed tomography identified diffuse ground-glass opacities with thickening interlobular septa (fig. 1). Bronchoalveolar lavage identified haemosiderin-laden macrophages. Based on

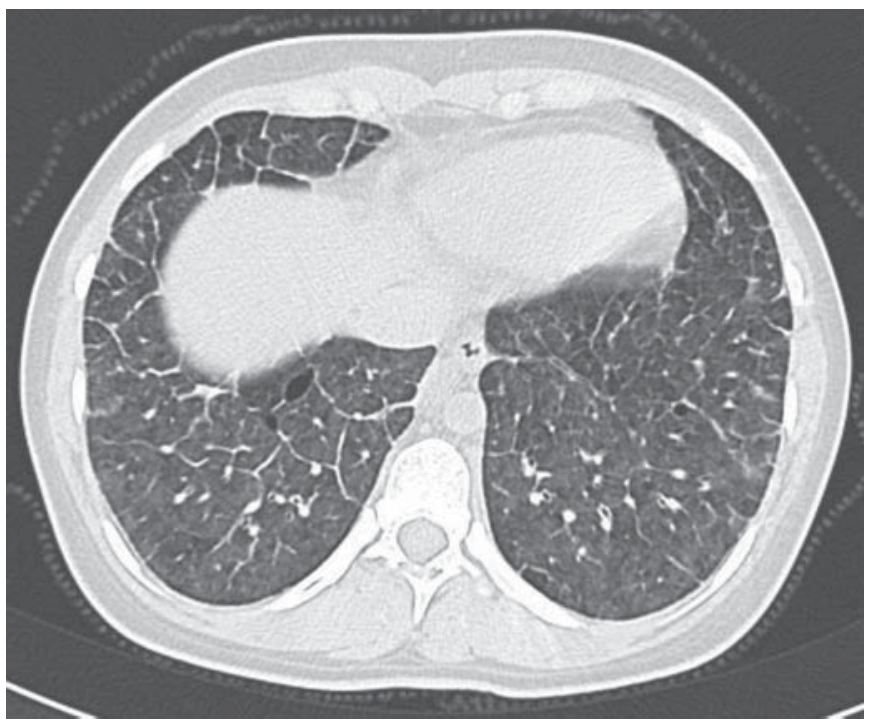

FIGURE 1. High-resolution computed tomography illustrating diffuse groundglass opacities with thickening interlobular septa. these findings a diagnosis of pulmonary veno-occlusive disease (PVOD) was hypothesised. Sildenafil was withdrawn and treatment with high-dose diuretics (oral furosemide $125 \mathrm{mg}$ b.i.d.) and cautious use of the endothelin-receptor antagonist bosentan (initial dose $62.5 \mathrm{mg}$ t.i.d., up-titrated to $125 \mathrm{mg}$ t.i.d.) was initiated. Lung transplantation was considered.

After 4 months of treatment an improvement in the patient's exercise capacity (6MWD $500 \mathrm{~m}$ ) and quality of life were observed, and supplemental oxygen was withdrawn. RHC showed a reduction in mean pulmonary arterial pressure, an increase in cardiac index and an improvement in systemic and mixed venous oxygen saturation (table 1 ). However, 1 yr later there was a progressive, slow deterioration in the patient's functional capacity and oxygen saturation, and inhaled iloprost $(2.5 \mu \mathrm{g}$ six times daily) was added to her treatment regimen with subjective improvement. Oxygen therapy was also initiated following deterioration in exercise capacity (6MWD $473 \mathrm{~m}$ ). Despite further subjective improvement, repeated RHC showed worsening haemodynamics with an increase in mean pulmonary arterial pressure, reduction in cardiac index and worsening mixed venous oxygen saturation.

In May 2008, the patient was recommended for a double lung transplant. In the period between May and December 2008 there was further deterioration in functional capacity and oxygen saturation, requiring an increase in her furosemide dose to $375 \mathrm{mg} \cdot \mathrm{day}^{-1}$. Her exercise capacity continued to decline (6MWD $347 \mathrm{~m}$ ). Double lung transplantation was performed on January 20, 2009. Histological analysis of explanted lung tissue showed an atherosclerotic plaque in the lower pulmonary artery, interstitial oedema, massive alveolar congestion, iron-laden macrophages, and fibrous thickening, obstruction and arterialisation of the septal and pre-septal pulmonary veins (fig. 2). There was no apparent capillary involvement.

Following transplantation, echocardiography showed normalisation of the patient's heart shape and movement of the interventricular septum. The patient is fine and without symptoms 4 months after transplantation.

\section{DISCUSSION}

PVOD is one of the more malignant forms of pulmonary hypertension, with an estimated 1-yr mortality of $72 \%$ [1]. Unlike PAH, which only affects the distal pulmonary arteries, PVOD preferentially involves septal veins and pre-septal venules. Despite this difference, patients with PVOD may present with very similar clinical symptoms and haemodynamic characteristics as patients with idiopathic PAH. Therefore, differential diagnosis of PVOD can be challenging and misdiagnosis is relatively common. Around $5-10 \%$ of patients originally diagnosed with idiopathic PAH are subsequently found to have PVOD [2], and a recent study of patients diagnosed with various forms of PAH who failed to respond to therapy observed that $86 \%$ actually had PVOD [3].

Patients with PVOD may have a heterogeneous clinical presentation; a high degree of clinical suspicion and appropriate diagnostic work-up are required for diagnosis. Although a definitive diagnosis relies on histological examination of lung biopsies, this procedure is particularly high risk in these patients. However, a number of diagnostic tests can confirm 

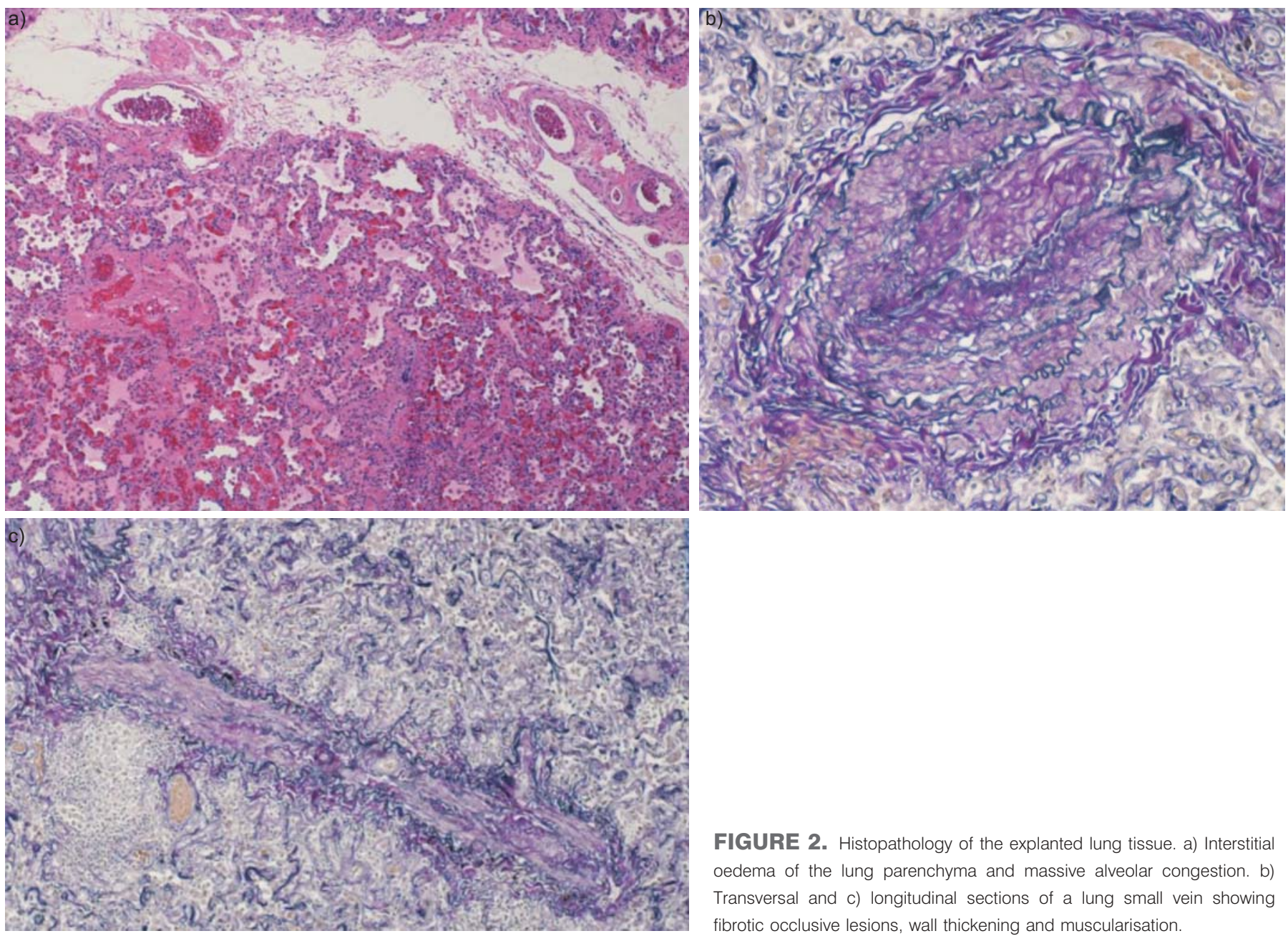

FIGURE 2. Histopathology of the explanted lung tissue. a) Interstitial oedema of the lung parenchyma and massive alveolar congestion. b) Transversal and c) longitudinal sections of a lung small vein showing fibrotic occlusive lesions, wall thickening and muscularisation.

the diagnosis of PVOD without the requirement for invasive techniques. Characteristic findings include centrilobular groundglass opacities, septal lines and mediastinal lymph node enlargement on high-resolution computed tomography of the chest, and the presence of haemosiderin-laden macrophages on bronchoalveolar lavage $[4,5]$. In addition, patients with PVOD have lower arterial oxygen tension at rest, lower oxygen saturation nadir during the 6-min walk test and lower diffusing lung capacity of carbon monoxide/alveolar volume than those with idiopathic PAH $[4,5]$.

PVOD is notoriously unresponsive to medical therapy and there are few treatment options other than lung transplantation for these patients. Clinical similarities suggest that PAH-specific therapies may be of use in these patients, but data are limited. A major concern with such treatment in PVOD is the development of pulmonary oedema, which can be potentially fatal. Pulmonary oedema has been reported with all classes of PAHspecific therapies including prostacyclins, calcium channel blockers, endothelin receptor antagonists and phosphodiesterase type-5 inhibitors, and may be most common with epoprostenol and calcium channel blockers [1, 4, 5]. Despite this, clinical improvement or stabilisation has been reported in a small number of patients treated with continuous intravenous prostacyclin [1, 6], oral sildenafil [7] and oral bosentan [8].
Recently, cautious use of continuous i.v. epoprostenol for 34 months was found to improve clinical and haemodynamic parameters in 12 patients with PVOD, suggesting that such therapy may be a useful bridge to lung transplantation [9]. Treatment of our patient with bosentan, in combination with high doses of furosemide to reduce lymphatic and venous congestion, may have contributed to improvements in her clinical status and may have slowed disease progression. Despite treatment, progressive deterioration continued, requiring eventual lung transplantation. Cautious use of specific drug treatments for $\mathrm{PAH}$, while not curative, may be useful as a bridging therapy to lung transplantation in patients with PVOD.

In conclusion, PVOD is a rare and severe form of pulmonary hypertension that presents a number of diagnostic challenges. Patients with PVOD may have a heterogeneous clinical presentation, requiring a high degree of clinical suspicion and robust diagnostic work-up. Misdiagnosis is relatively common. Accurate diagnosis is essential for appropriate management, especially since some PAH-specific therapies may be harmful in patients with PVOD. Although lung transplantation is the treatment of choice for patients with PVOD, the use of specific therapies for $\mathrm{PAH}$ in addition to high doses of diuretics may be helpful in some patients, and merits further evaluation. 


\section{STATEMENT OF INTEREST}

None declared.

\section{ACKNOWLEDGEMENTS}

The authors received editorial assistance from Elements Communications Ltd (Westerham, UK), supported by Actelion Pharmaceuticals Ltd (Allschwil, Switzerland).

\section{REFERENCES}

1 Holcomb BW Jr, Loyd JE, Ely EW, et al. Pulmonary veno-occlusive disease: a case series and new observations. Chest 2000; 118: 1671-1679.

2 Mandel J, Mark EJ, Hales CA. Pulmonary veno-occlusive disease. Am J Respir Crit Care Med 2000; 162: 1964-1973.

3 Harch S, Whitford H, McLean C. Failure of medical therapy in pulmonary arterial hypertension: is there an alternative diagnosis? Chest 2009; 135: 1462-1469.
4 Montani D, Achouh L, Dorfmüller $\mathrm{P}$, et al. Pulmonary venoocclusive disease: clinical, functional, radiologic, and hemodynamic characteristics and outcome of 24 cases confirmed by histology. Medicine (Baltimore) 2008; 87: 220-233.

5 Montani D, Price LC, Dorfmuller P, et al. Pulmonary veno-occlusive disease. Eur Respir J 2009; 33: 189-200.

6 Okumura H, Nagaya N, Kyotani S, et al. Effects of continuous IV prostacyclin in a patient with pulmonary veno-occlusive disease Chest 2002; 122: 1096-1098.

7 Barreto AC, Franchi SM, Castro CR, et al. One-year follow-up of the effects of sildenafil on pulmonary arterial hypertension and venoocclusive disease. Braz J Med Biol Res 2005; 38: 185-195.

8 Barboza CE, Jardim CV, Hovnanian AL, et al. Pulmonary venoocclusive disease: diagnostic and therapeutic alternatives. J Bras Pneumol 2008; 34: 749-752.

9 Montani D, Jaïs X, Price LC, et al. Cautious use of epoprostenol therapy is a safe bridge to lung transplantation in pulmonary venoocclusive disease. Eur Respir I 2009; [Epub ahead of print PMID 19541723]. 